\title{
A note on the cumulative and the accumulated hazard function
}

\author{
Dejan Škanata \\ Enconet d.o.o., Zagreb \\ University of Applied Sciences Velika Gorica, Velika Gorica \\ Faculty of Electrical Engineering and Computing, University of Zagreb \\ Zagreb, Croatia
}

\begin{abstract}
In probability and statistics, reliability theory and survival analysis, there exists a 20-year-old dilemma, initially raised by L.M. Leemis, on whether the cumulative or the accumulated hazard function in the discrete domain is more appropriate to be used in various types of applications. Here, we propose that priority should be given to the accumulated hazard function.
\end{abstract}

Keywords-accumulated hazard function, cumulative hazard function, exponential distribution, geometric distribution, hazard function in average, memoryless, Weibull distribution

\section{INTRODUCTION}

The cumulative hazard function (also known as the integrated hazard function or the hazard potential) is one of the several functions describing lifetime. It is a nondecreasing function of time which does not have probabilistic connotation, meaning there is no law of probability that leads to the cumulative hazard function. Yet it plays a key role in reliability and survival analysis. In the discrete domain two such functions are recognized, the cumulative and the accumulated hazard function.

The first mention of the dilemma of defining the cumulative hazard function in the discrete domain was, as far as we are aware, introduced by Leemis in [1]. He underlines that two possible, but different choices exist. The first definition (cumulative) parallels the relationship established in the continuous domain while the second definition accumulates the hazard function as it evolves over time. Leemis also underlined that two functions are very close when probability mass function values are small. However, the example he supplied he applied the first definition.

Lawless [2] also noted that by analogy with continuous case the cumulative hazard function could be defined in two different ways in the discrete domain. In a section of his book in which he introduces a way to unify continuous, discrete and mixed lifetime distributions in one framework, he adopted the second definition.
Kemp [3] repeats the dilemma recognized by Leemis and defines the cumulative $\Lambda_{t}$, and the accumulated hazard function $H_{t}$, in the following manner:

$$
\begin{aligned}
& \Lambda_{t}=-\ln \left(R_{t}\right) \\
& H_{t}=\sum_{j=0}^{t} h_{j}
\end{aligned}
$$

where $R_{t}$ is the reliability function and $h_{j}$ is the hazard function.

Kemp also establishes the relationships between two functions in the following way:

$$
\begin{aligned}
& \Lambda_{t}=-\sum_{j=0}^{t-1} \ln \left(1-H_{j}+H_{j-1}\right) \\
& H_{t}=\sum_{j=0}^{t}\left(1-e^{\Lambda_{j}-\Lambda_{j+1}}\right)
\end{aligned}
$$

and emphasizes that $\Lambda_{t} \neq H_{t}$.

Lai and Xie [4] also emphasize that $H_{t}$ is not equivalent to $\Lambda_{t}$ and underline that the mentioned nonequivalence has been noted by several authors. Also they noted that this fact has prompted some authors to seek for an alternative definition of the hazard function. Lai and Xie dedicate a chapter of their excellent book to this topic by quoting and explaining to some extent the works of Roy and Gupta [5], Bracquemond et al. [6] and Xie et al. [7].

Rinne [8] iterates Leemis's and Kemp's findings and establishes the complete set of equations describing relationships between the most popular discrete lifetime representations such as the probability mass function, reliability function, hazard function, cumulative hazard function, accumulated hazard function and the mean residual life function. Rinne stresses the alternative definition of the hazard function called the pseudo-hazard rate given by Roy and Gupta [5]. Also Rinne makes a note on Cox and Oakes [9] somewhat different definition of the cumulative hazard function for discrete lifetimes and concludes that if $h_{t}$ is small, the Cox and Oakes definition is reducing approximately to $H_{t}$.

\section{DiLEMMA Formulation}

It should be summarized here that both previously mentioned discrete definitions recognized by Leemis and by other authors afterwards, are based on the analogy to the 
continuous domain. The first definition is based on the relationship analogy (logarithm) while the second definition is based on the mathematical operation analogy (switching integration with summation) as it is shown in Table I.

Table I. Continuous vs. discrete definitions

\begin{tabular}{|c|c|}
\hline Continuous domain & Discrete domain \\
\hline$H(t)=-\ln [R(t)]$ & $\Lambda_{t}=-\ln \left(R_{t}\right)$ \\
\hline$H(t)=\int_{o}^{t} h(\tau) d \tau$ & $H_{t}=\sum_{j=0}^{t} h_{j}$ \\
\hline
\end{tabular}

In the continuous domain there is a unique cumulative function no matter if it is expressed by the reliability function, $R(t)$, or by the hazard function, $h(t)$. It means that in the continuous domain the following identity is valid:

$$
-\ln [R(t)]=\int_{o}^{t} h(\tau) d \tau
$$

In the discrete domain this is not so. Namely, functions $\Lambda_{t}$ (cumulative) and $H_{t}$ (accumulated) are somewhat different as shown by Kemp:

$$
-\ln \left(R_{t}\right) \neq \sum_{j=0}^{t} h_{j}
$$

The Leemis dilemma may be formulated by posing the following question: Which of the two discrete hazards functions, cumulative or accumulated, is more credible to be used in various types of applications? The question may be asked in the following way as well: Which one of two discrete definitions is more similar to the continuous case?

\section{General CONSIDERATIONS}

The difference between $\Lambda_{t}$ and $H_{t}$ starts at the very beginning, with $t=0$. At point $t=0$ the following is valid for the two functions:

- $\Lambda_{0}=0$ because $R_{0}$ equals 1 by definition just as in the continuous domain, and

- $H_{0}=h_{0}$ where $h_{0}$ may take values between 0 and 1 . This is because the hazard function in the discrete domain is defined strictly as conditional probability while in continuous domain the hazard function has an approximate meaning of conditional probability, Singpurwalla [10].

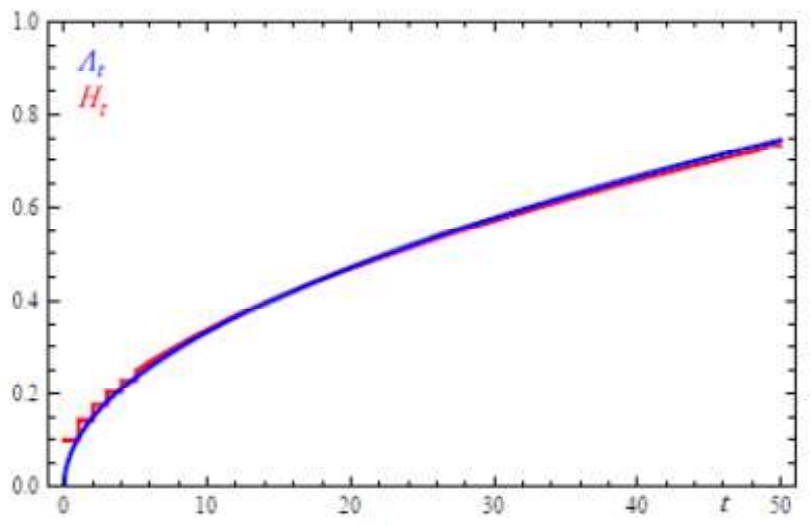

Generally, $\Lambda_{t}$ increases faster than $H_{t}$ with $t$ and after some time $\Lambda_{t}$ exceeds $H_{t}$. This means that exists a virtual point where two functions cross. Relation between $\Lambda_{t}$ and $H_{t}$ depends upon the value of $p$. For small enough $p, \Lambda_{t}$ and $H_{t}$ after the initial dichotomy, become very close, meaning the virtual cross-point appears much later. Higher the value of $p$, the sooner the virtual cross-point appears, after which $\Lambda_{t}$ and $H_{t}$ diverge.

The above statements are illustrated in the example of the geometric distribution. For this distribution the virtual crosspoint, $T_{v c p}$, can be calculated by applying the following formula:

$$
T_{v c p}=-\frac{p}{p+\ln (1-p)}
$$

which is displayed in Fig. 1.

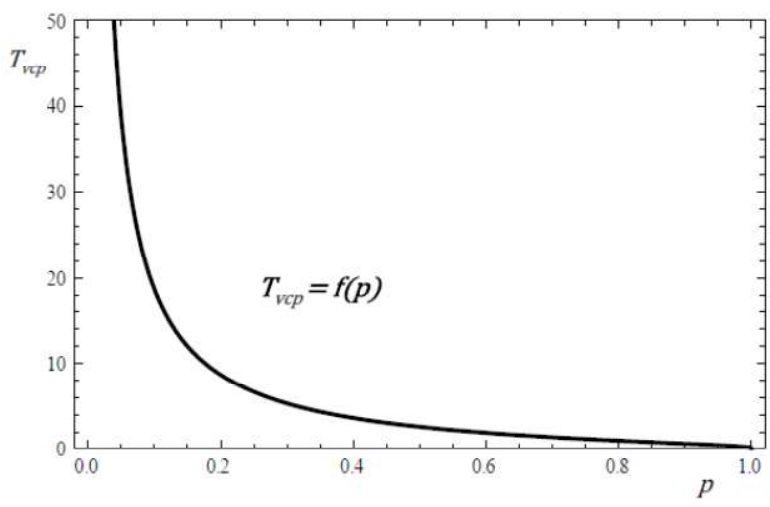

Fig. 1 Virtual cross points of the geometric distribution

It is obvious from the above that for small $p, T_{v c p}$ occurs at higher values ( $\Lambda_{t}$ and $H_{t}$ are close) while for high $p, T_{v c p}$ occurs at lower values $\left(\Lambda_{t}\right.$ and $H_{t}$ diverge).

Very similar results are obtained for the discrete Weibull distribution with shape parameter $\beta$. Fig. 2 and Fig. 3 show hazard functions with $0<\beta<1$ (non-increasing hazard function) and $\beta>1$ (non-decreasing hazard function) of the Weibull distribution, respectively. While for $\Lambda_{t}$ analytic form exists, $H_{t}$ is obtained numerically.

Fig. $2 \Lambda_{t}$ (blue) and $H_{t}$ (red) functions associated to the discrete Weibull distribution with $\beta=0.5$ and $p=0.1$ (left panel), and with $\beta=0.5$ and $p=0.8$ (right panel) 

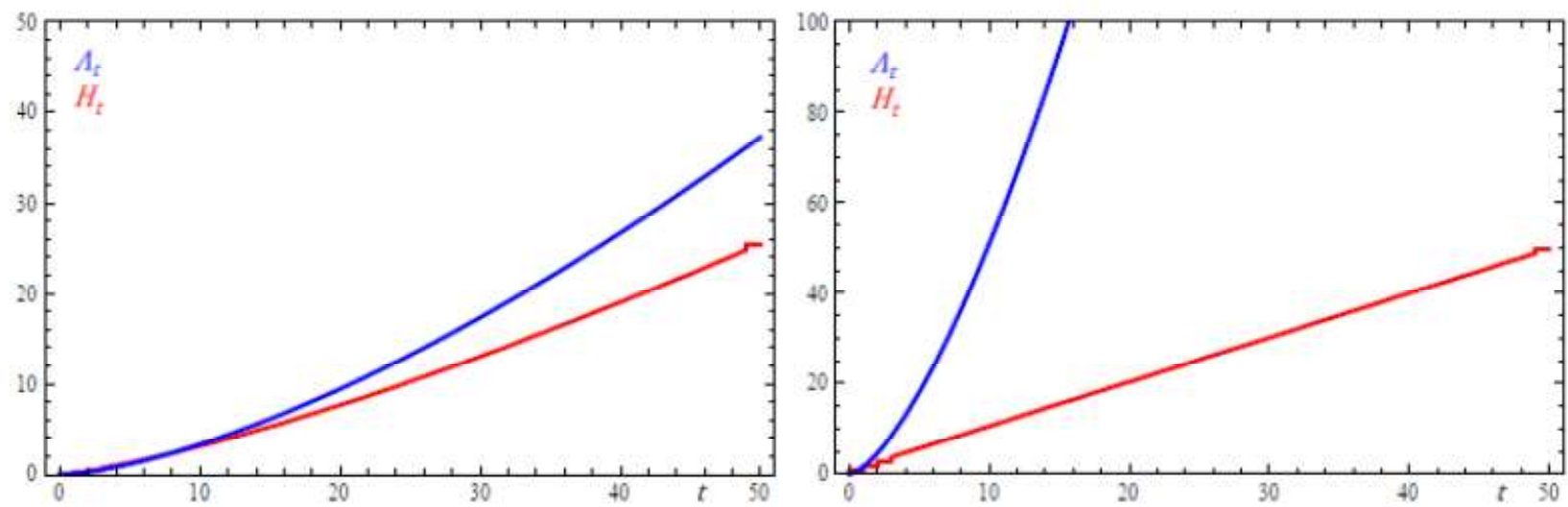

Fig. $3 \Lambda_{t}$ (blue) and $H_{t}$ (red) functions associated to the discrete Weibull distribution with $\beta=1.5$ and $p=0.1$ (left panel), and with $\beta=1.5$ and $p=0.8$ (right panel)

From the above figures it is evident that in case of small $p$ and non-increasing $h(t)$ functions $\Lambda_{t}$ and $H_{t}$ are close except at $t=0$ (Fig. 2, left panel). Contrary, in case of high $p$ and non-decreasing $h(t)$ two functions diverge significantly (Fig. 3 , right panel).

\section{MEMORYLESS DISTRIBUTIONS}

Here we include a short discussion on the major properties of exponential and geometric distributions, which will become important for the main result of the paper. It is wellknown that the exponential distribution is the only memoryless continuous distribution and the geometric distribution is the only memoryless discrete distribution. The primary effect of the memoryless property is that the hazard function is constant in time. Consequently, for these two distributions the following applies:

$$
\begin{aligned}
& h(t)=\lambda \\
& h_{t}=p
\end{aligned}
$$

where $\lambda>0$ is the scale parameter of the exponential distribution and $0<p \leq 1$ is the success probability of the geometric distribution.

We involve in this discussion the continuous and discrete hazard functions in average, $h^{*}(t)$ and $h_{t}^{*}$. This function is usually abbreviated in reliability literature as FRA (failure rate in average).

In the case of exponential distribution $h^{*}(t)$ strictly follows $h(t)$ function. This is because $h^{*}(t)$ is defined as:

$$
h^{*}(t)=\frac{H(t)}{t}
$$

The substitution (see Table I.) gives the following:

$$
h^{*}(t)=\frac{-\ln [R(t)]}{t}=\frac{-l\left[e^{-\lambda t}\right]}{t}=\lambda=h(t)
$$

where $R(t)=e^{-\lambda t}$ is the reliability (survivor) function of the exponential distribution.

We further claim that the effects of the memoryless property are unique and irrespective to the domain considered, continuous or discrete. This claim implies that in case of the geometric distribution $h_{t}^{*}$ function must strictly follow $h_{t}$ function as well. It is easy to show that such condition satisfies $H_{t}$ and not $\Lambda_{t}$ function. Namely, for the geometric distribution,

$$
p_{t}=p(1-p)^{t} ; t=0,1,2,3 \ldots
$$

by applying equations given in Table I. the following is obtained:

$$
\begin{aligned}
& h_{H_{t}}^{*}=\frac{H_{t}}{t+1}=\frac{\sum_{j=0}^{t} p}{t+1}=\frac{p(t+1)}{t+1}=p=h_{t} \\
& h_{\Lambda_{t}}^{*}=\frac{\Lambda_{t}}{t+1}=\frac{-l\left(R_{t}\right)}{t+1}=\frac{-\ln \left[(1-p)^{t}\right]}{t+1} \neq h_{t}
\end{aligned}
$$

and for the shifted geometric distribution,

$$
p_{t}=p(1-p)^{t-1} ; t=1,2,3 \ldots
$$

the following applies:

$$
\begin{aligned}
& h_{H_{t}}^{*}=\frac{H_{t}}{t}=\frac{\sum_{j=1}^{t} p}{t}=\frac{p t}{t}=p=h_{t} \\
& h_{\Lambda_{t}}^{*}=\frac{\Lambda_{t}}{t}=\frac{-\ln \left(R_{t}\right)}{t}=\frac{-\ln \left[(1-p)^{t-1}\right]}{t} \neq h_{t}
\end{aligned}
$$

\section{WEIBULl DiSTRIBUTION}

We apply the same approach to Weibull continuous and discrete distributions. The difference is that $h^{*}(t)$ function by its shape not strictly but faithfully follows $h(t)$ function. Term "faithfully follows" in this context means that both functions start at the same point and approach the same limit. Also we find that the ratio of two functions is fixed and equal to $\beta$ (shape parameter of the Weibull distribution):

$\frac{h(t)}{h^{*}(t)}=\beta$

For the continuous Weibull distribution this is shown in Fig. 4. It should be noted that $h^{*}(t)$ function has slower decline (left panel), i.e. has slower growth (right panel) when compared to $h(t)$. 

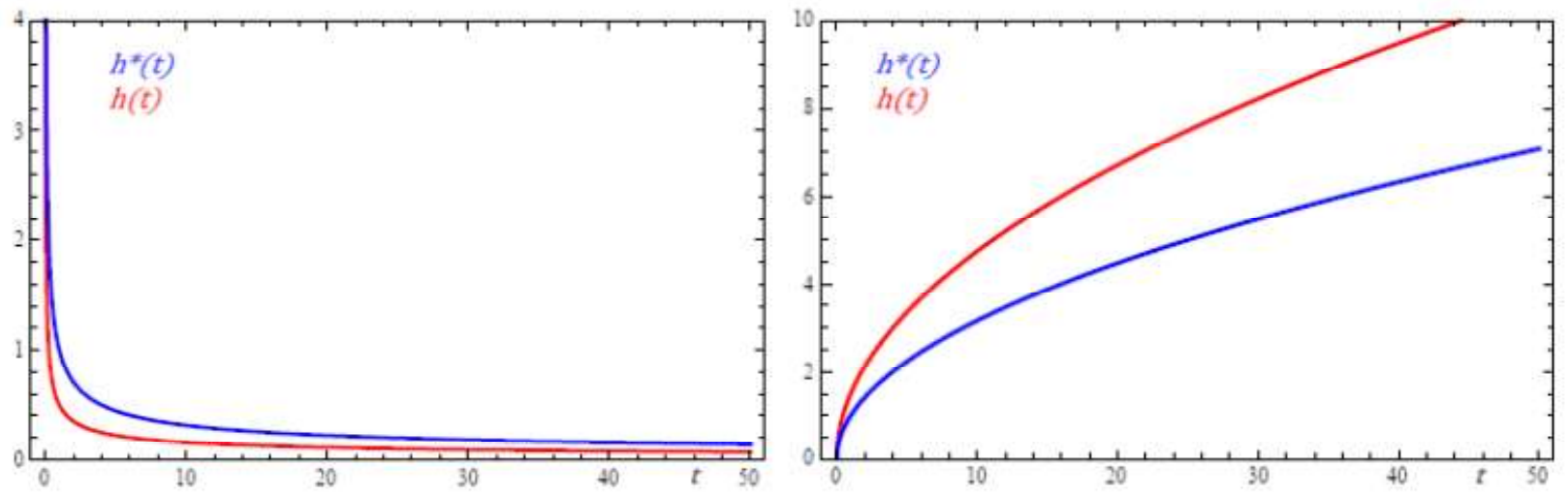

Fig. $4 h(t)$ (red) and $h^{*}(t)$ (blue) functions of the continuous Weibull distribution with $\beta=0.5$ and $\lambda=1$ (left panel), and with $\beta=1.5$ and $\lambda=1$ (right panel)

For the discrete Weibull distribution results are shown in Figures 5 and 6 . Note that in this case the ratio $\frac{h_{t}}{h_{H_{t}}^{*}}$ is very close to $\beta$ while the ratio $\frac{h_{t}}{h_{\Lambda_{t}}^{*}}$ differs from $\beta$ significantly.
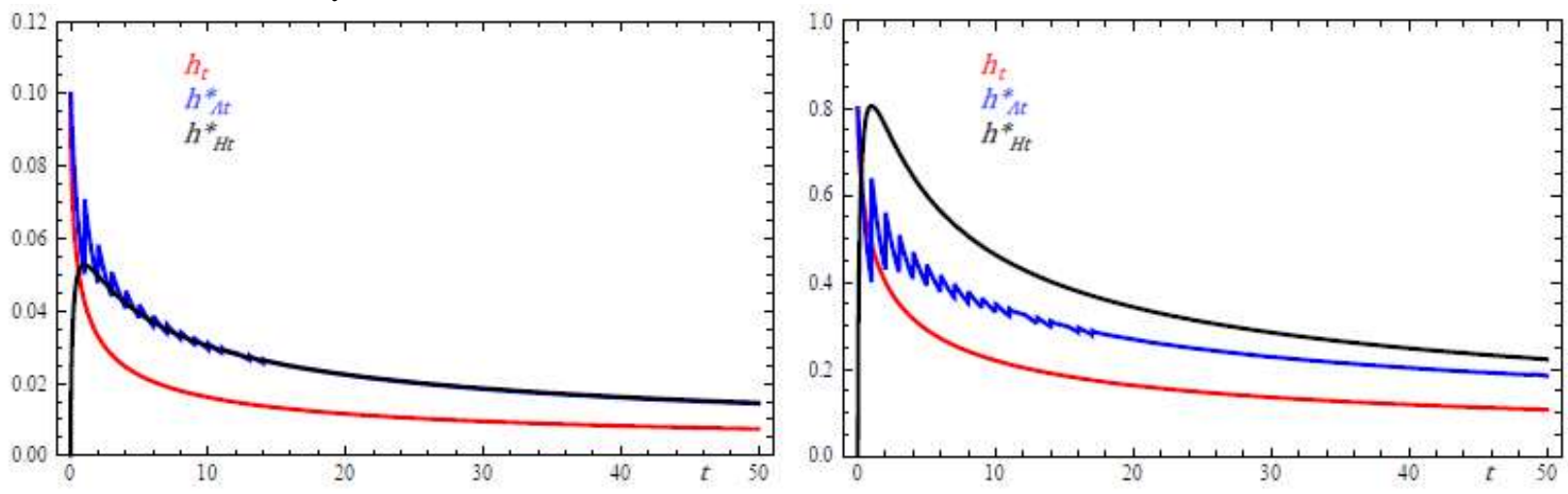

Fig. $5 h_{t}$ (red), $h_{\Lambda_{t}}^{*}$ (blue) and $h_{H_{t}}^{*}$ (black) functions of the discrete Weibull distribution with $\beta=0.5$ and $p=0.1$ (left panel), and with $\beta=0.5$ and $p=0.8$ (right panel)
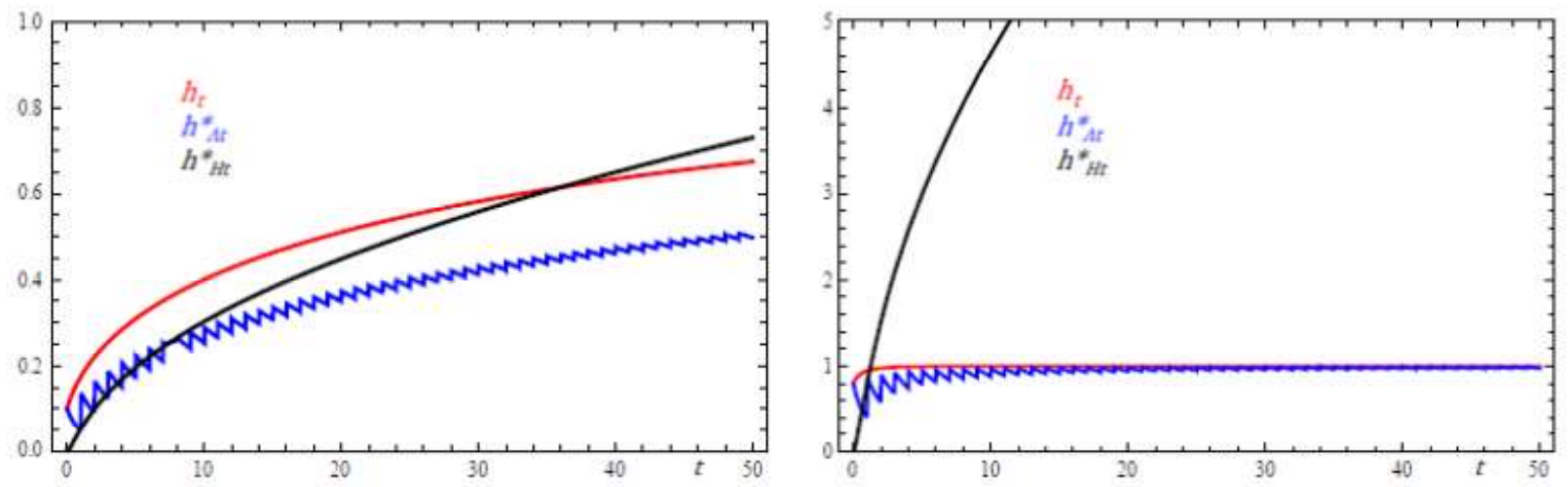

Fig. $6 h_{t}$ (red), $h_{\Lambda_{t}}^{*}$ (blue) and $h_{H_{t}}^{*}$ (black) functions of the discrete Weibull distribution with $\beta=1.5$ and $p=0.1$ (left panel) and with $\beta=1.5$ and $p=0.8$ (right panel)

Based on Fig. 5 and Fig. 6 for the discrete Weibull distribution the following may be concluded:

- for the non-increasing case of $h_{t}(0<\beta<1)$ and small $p$, after the initial dichotomy, the functions $h_{\Lambda_{t}}^{*}$ and $h_{H_{t}}^{*}$ become very close and they may be approximated (Fig. 5, left panel)

- in other cases the difference between two functions become more significant. In particular in the case of nondecreasing $h_{t}(\beta>1)$ and high value of $p$. In such a case 
(Fig. 6, right panel), function $h_{\Lambda_{t}}^{*}$ gets values above 1 which in the discrete domain make no sense.

Therefore for the discrete Weibull distribution, we prefer usage of the accumulated hazard function instead of the cumulative hazard function.

\section{CONCLUSION}

The analysis presented here shows that a priority in the discrete domain should be given to the accumulated hazard function, as $H_{t}$ function has more similar properties to the cumulative hazard function as defined in the continuous domain.

By applying $H_{t}$ function, the same memoryless effect is obtained in discrete domain (geometric distribution) as in continuous domain (exponential distribution). The similarity criterion also favors the accumulated hazard function in case of the discrete Weibull distribution. In the case of small $p$ and non-increasing hazard function $(0<\beta<1)$ of the Weibull distribution, the two functions provide almost identical information, except at $t=0$. In other cases the accumulated hazard function seems to be more credible. If the results for the discrete Weibull distribution can be assumed representative, this shows that defining the cumulative hazard function based on the analogy between mathematical operations (switching integration with summation) is likely correct.

Finally, these examples show a potential to resolve the Leemis dilemma. We are also confident that the alternative definitions of discrete hazard functions, such as pseudohazard rate, are not needed for this purpose.

\section{ACKNOWLEDGMENTS}

The Leemis dilemma has emerged as an interesting topic within the Risk Analysis course held by the author at the Faculty of Electrical Engineering and Computing, University of Zagreb. This paper is a response to the many questions posed by his students.

\section{References}

[1] L.M. Leemis, Reliability: Probabilistic Models and Statistical Methods, Prentice-Hall, 1995.

[2] J.F. Lawless, Statistical Models and Methods for Lifetime Data, Second Edition, Wiley-Interscience, 2003.

[3] A.W. Kemp, Classes of Discrete Lifetime Distributions, Communications in Statistics, Theory and Methods, Vol. 33, No. 12, pp. 3069-3093, 2004.

[4] C.D. Lai and M. Xie, Stochastic Ageing and Dependence for Reliability, Springer, 2006.

[5] D. Roy and R.P. B. Gupta, Characterizations and model selections through reliability measures in the discrete case, Statistics and Probability Letters, 43, pp. 197-206, 1999.

[6] C. Bracquemond, O. Gaudoin, D. Roy and M. Xie, On some notions of discrete ageing. In Y. Hayakawa, T. Irony and M. Xie (Editors), System and Bayesian Reliability: Essays in Honor of Professor Richard E. Barlow on His 70th Birthday, Vol 5, Series on Quality, Reliability \& Engineering Statistics, pp. 185-197, World Scientific Press, 2001.

[7] M. Xie, O. Gaudoin and C. Bracquemond, Redefining failure rate function for discrete distributions, International Journal of Reliability, Quality and Safety Engineering, 9(3), pp. 275-285, 2002.

[8] H. Rinne, The Hazard Rate, Theory and Inference, With supplementary MATLAB-Programs, Justus-LiebigUniversity, 2014.

[9] D.R. Cox and D. Oakes, Analysis of Survival Data, Chapman \& Hall, 1984.

[10] N.D. Singpurwalla, The Hazard Potential: Introduction and Overview, Journal of the American Statistical Association, Vol. 101, No. 476, pp. 1705-1717, 2006. 